\title{
Preface
}

\section{Telemedicine in Intensive Care Units: A Luxury or Necessity?}

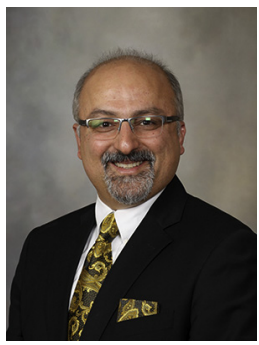

Kianoush B. Kashani, MD, MS Editor

The advent of telemedicine programs and their utilization in intensive care units (ICU), started in the 1970s, was initially designed to enhance the ability of local providers by simple verbal or video consultation with an ICU expert. Over the course of years with evolution of clinical informatics and connectivity, the idea has grown to the contemporary Tele-ICU practices, which not only provide expert opinion to the local providers but also offer a wide range of services, including monitoring, supervisions, communications with patients, families, and local providers of many disciplines, active resuscitation and stabilization, optimization, and deescalation processes for critically ill patients in shock, and additional services, including Tele-pharmacy, Tele-dialysis, Tele-stroke, and so forth.

Over the course of the past four decades, one can witness the progressive use of more modern and advanced technological tools to provide appropriate care to the patients at need. This not only includes better communication tools and video-connectivity devices but also the use of more advanced artificial intelligence and clinical decision support systems, which has provided a tremendous opportunity to provide safer and more efficient and timely care. While some use Tele-ICU services to enhance the safety of their own local ICU practices, the majority of organizations offer such services to the remote ICUs that have limited availabilities of experts throughout the day and week. This has opened a whole new horizon for the ability of clinicians to provide high-quality critical care in underserved areas throughout the globe.

Building an infrastructure of Tele-ICU and maintaining it are not minuscule tasks, and barriers like legal hurdles, need for accreditation, and appropriate training and licensing could limit the spectrum of what could be done using Tele-ICU. Having said that, the growth of several active and effective Tele-ICU programs has paved the way for other institutions to offer or receive such services. One crucial 
administrative aspect of Tele-ICU is constant monitoring for cost-effectiveness of services to enhance the viability of these programs.

One of the essential aspects of Tele-ICU is the need for growing knowledge regarding the best practices to improve outcomes and provider, receiver, patient, and family satisfaction. Therefore, focusing on Tele-ICU-centered research and quality improvement projects is essential in providing ever-improving care to the most vulnerable patients in ICUs. Innovative projects to employ new technologies for care enhancement are an absolutely necessary to allow the practice of Tele-ICU to grow beyond the current level of sophistication.

The other important factor to be considered is the multidisciplinary nature of TeleICU care. The successful Tele-ICU provider programs (Hub) are those that have been able to make and maintain teams of nurses, pharmacists, advanced clinical practitioners, respiratory therapists, information technologists, secretaries, and intensive care and other subspecialty physicians that provide services to multiple disciplines in the receiving (Spoke) ICUs. Without appropriate organization and communication, often the care would not be optimized, which increases the chances of harm and missed opportunities and increases cost.

Critical Care Clinics recognizes the importance of this new path for providing appropriate care to ICU patients and hence has dedicated this issue to the topic of Tele-ICU. In this issue of Critical Care Clinics, several world experts in the field share their vast knowledge and experience regarding different aspects of Tele-ICU programs. We start with the administrative and legal requirements for initiation and maintenance of a Tele-ICU program, including accreditation processes. Then, we focus on the current state and future of technological aspects of successful Tele-ICU programs before concentrating on the organization of optimum teams and models of care that could be offered by Tele-ICU. We then report the impact of Tele-ICU on critically ill patient outcomes, how to maintain the quality of care, patient/provider satisfaction, and cost-effectiveness of Tele-ICU programs. We also describe some of the recently described innovations and recognized limitations of these programs along with a variety of services that could be provided using the Tele-ICU infrastructure. This issue offers a wide range of ideas for future practice improvements and research projects, which it is hoped translates to improved outcomes of ICU patients' recipient of these services.

Kianoush B. Kashani, MD, MS Division of Nephrology and Hypertension

Division of Pulmonary and

Critical Care Medicine

Department of Medicine

Mayo Clinic

200 First Street Southwest Rochester, MN 55905, USA

E-mail address: 